\title{
Contributions of village animal health workers to foot-and-mouth disease control in Cambodia
}

\author{
Socheat Sieng ${ }^{1}$, Ian Patrick ${ }^{2}$, P.A. Windsor ${ }^{3}$, Stephen Walkden-Brown ${ }^{4}$, James Kerr ${ }^{5}$, \\ Sovann $\mathrm{Sen}^{6}$, C. Sar ${ }^{6}$, Robert $\mathrm{Smith}^{7}$, and Reatrey Kong ${ }^{8}$ \\ ${ }^{1}$ General Directorate of Animal Health and Production Phnom Penh Cambodia \\ ${ }^{2}$ University of Liverpool \\ ${ }^{3}$ The University of Sydney School of Veterinary Science \\ ${ }^{4}$ University of New England School of Environmental and Rural Science \\ ${ }^{5}$ Hunter Local Land Services Paterson NSW Australia \\ ${ }^{6}$ Ministry of Agriculture Forestry and Fisheries Phnom Penh Cambodia \\ ${ }^{7}$ Southern Cross University School of Environment Science and Engineering \\ ${ }^{8}$ Independent Livestock consultant Pursat Cambodia
}

May 12, 2021

\begin{abstract}
Local animal health services in rural communities are mainly provided by village animal health workers (VAHW), although the participation and contribution of VAWHs to disease prevention is uncertain. To address this, a desktop review of national VAWH data between 2011 - 2020 also conducted in December 2020, supporting a detailed survey on the involvement of VAHWs in disease prevention programs conducted between February to March 2014. The survey used guided group discussion with VAHWs $(n=198)$ from the two Cambodian provinces of Kampong Cham and Pursat. This study identified that VAHWs generated less than $22 \%$ of their annual household incomes from animal health services. Less than one-third had vaccinated livestock against FMD, with none having vaccinated cattle every six months during the study period, and nearly half of the VAHWs having never vaccinated their own cattle against FMD. As no privately-provided FMD vaccination services occurred in these communities, with all vaccines delivered through the government-subsidised program, the findings confirmed that VAHWs only vaccinated animals against FMD when vaccines were made available by the Government. The desktop review found that the number of VAHWs in 2020 declined by more than $24 \%$ since 2017 and the proportion of female VAHWs was consistently low, with a mean of 8.26 ( \pm 1.019$)$. These findings confirm there are considerable weaknesses in the VAHW system in Cambodia, particularly in contributing to FMD control. Cambodian animal health authorities require more effective policies to strengthen the current VAHW system, improving: their services delivery; their retention as 'active'; their development of more sustainable roles with lower 'dropout' rates; and the prolonged gender inequity. With the limited availability of government-subsidised FMD vaccination currently, extension programs that engage VAHWs and farmers in seeking privately funded and delivered FMD vaccination that incorporates appropriate multivalent FMD serotype vaccines of high quality, delivered in small dose vials from a robust cold chain, is suggested. This strategy would assist VAHWs to contribute to the provision of private livestock vaccination services that are likely essential for sustainable FMD prevention and control in Cambodia.
\end{abstract}

Contributions of village animal health workers to foot-and-mouth disease control in Cambodia S. Sieng ${ }^{1}$, I.W. Patrick ${ }^{2,3}$, P.A. Windsor ${ }^{4}$, S.W. Walkden-Brown ${ }^{5}$, J. Kerr ${ }^{6}$, S. Sen ${ }^{7}$, C. Sar ${ }^{7}$, R.G.B. Smith ${ }^{8}$, R Kong9

${ }^{1}$ General Directorate of Animal Health and Production, Phnom Penh, Cambodia 
${ }^{2}$ University of Liverpool, UK

${ }^{3}$ ARECS P/L, Armidale, NSW, Australia

${ }^{4}$ Sydney School of Veterinary Science, The University of Sydney, Camden, NSW, Australia

${ }^{5}$ Animal Science, School of Environmental and Rural Science, University of New England, Armidale, NSW, Australia

${ }^{6}$ Hunter Local Land Services, Paterson, NSW, Australia

${ }^{7}$ Ministry of Agriculture, Forestry and Fisheries, Phnom Penh, Cambodia

${ }^{8}$ School of Environment, Science and Engineering, The Southern Cross University, Lismore, NSW, Australia

${ }^{9}$ Independent Livestock consultant, Pursat, Cambodia

Correspondence:

S. Sieng, General Directorate of Animal Health and Production, Phnom Penh, Cambodia.

Tel: +855 89908917 or +61401512627 ; s.socheat2010@gmail.com

\section{Abstract}

Local animal health services in rural communities are mainly provided by village animal health workers (VAHW), although the participation and contribution of VAWHs to disease prevention is uncertain. To address this, a desktop review of national VAWH data between 2011 - 2020 also conducted in December 2020, supporting a detailed survey on the involvement of VAHWs in disease prevention programs conducted between February to March 2014. The survey used guided group discussion with VAHWs $(\mathrm{n}=198)$ from the two Cambodian provinces of Kampong Cham and Pursat. This study identified that VAHWs generated less than $22 \%$ of their annual household incomes from animal health services. Less than one-third had vaccinated livestock against FMD, with none having vaccinated cattle every six months during the study period, and nearly half of the VAHWs having never vaccinated their own cattle against FMD. As no privately-provided FMD vaccination services occurred in these communities, with all vaccines delivered through the governmentsubsidised program, the findings confirmed that VAHWs only vaccinated animals against FMD when vaccines were made available by the Government. The desktop review found that the number of VAHWs in 2020 declined by more than $24 \%$ since 2017 and the proportion of female VAHWs was consistently low, with a mean of $8.26( \pm 1.019)$. These findings confirm there are considerable weaknesses in the VAHW system in Cambodia, particularly in contributing to FMD control. Cambodian animal health authorities require more effective policies to strengthen the current VAHW system, improving: their services delivery; their retention as 'active'; their development of more sustainable roles with lower 'dropout' rates; and the prolonged gender inequity. With the limited availability of government-subsidised FMD vaccination currently, extension programs that engage VAHWs and farmers in seeking privately funded and delivered FMD vaccination that incorporates appropriate multivalent FMD serotype vaccines of high quality, delivered in small dose vials from a robust cold chain, is suggested. This strategy would assist VAHWs to contribute to the provision of private livestock vaccination services that are likely essential for sustainable FMD prevention and control in Cambodia.

Key words : animal health services; Cambodia; cattle; foot and mouth disease; village animal health worker; vaccination

\section{Introduction}

The Kingdom of Cambodia is a Southeast Asian (SEA) country of $181,035 \mathrm{~km}^{2}$ that shares borders with the Lao People's Democratic Republic (PDR) in the north, Thailand in the north and west, and Vietnam to the south and east. Cambodia consists of 24 provinces and one municipality, with a population estimated to be about 16.9 million (World Population Review, 2021). Agriculture is integral to the life of smallholder farmers in rural communities, with cattle-raising important, providing a source of income, asset storage, manure for 
fertiliser, transport and on some farms, draught power (Young et al., 2014b). Cattle-raising is often operated alongside other activities, particularly rice and crop production and other off-farm activities (Samkol, Sath, Patel, Windsor, \& Holtenius, 2015; Young et al., 2014b).

Animal disease outbreaks pose significant threats to livestock sectors in Cambodia, both from the economic impacts of the disease and the measures required to reduce disease introduction risk. FMD is recognised as the most significant transboundary animal diseases (TADs) affecting large ruminants in the Mekong region of SEA and beyond, particularly Cambodia (Blacksell, Siengsanan-Lamont, Kamolsiripichaiporn, Gleeson, \& Windsor, 2019). FMD causes significant financial impacts in Cambodia, both at smallholder farmer households (Young et al., 2013b) and the national levels (Young et al., 2014c). Despite many years of attempted FMD control by individual countries assisted by regional coordination through the SEACFMD program of the World Organisation for Animal Health (OIE) office in Bangkok, numerous factors have prevented the successful control of FMD within the region. These include 'informal' transboundary movement of livestock and their products, challenges in implementing vaccination programmes, the circulation of multiple and emerging virus topotypes and lineages, low-level technical capacity and biosecurity of veterinary services at national levels, limited farmer knowledge of FMD management, failures in the timely reporting and response to outbreaks, and limitations in national and international FMD control programmes (Blacksell et al., 2019). In Cambodia, a survey of smallholder farmers $(n=300)$ found that FMD outbreaks occurred every year during the study period, with a morbidity rate of over 30\% (Sieng et al., 2021a). Biosecurity and vaccination practices were inadequate and it was concluded that poor knowledge of disease transmission and biosecurity, with low FMD vaccination coverage and a focus on treatment, contributed to regular FMD outbreaks in these communities.

Due to the lack of veterinary personnel and officials at the district and provincial levels, the Cambodian veterinary authority's significant task was to provide a para-veterinary animal health service enabling most farmers to access basic veterinary services when required. Para-veterinarians are considered crucial in providing front-line veterinary care and advice to smallholder farmers in countries with under-resourced national veterinary services, although often these individuals undergo basic training with minimal monitoring and evaluation of performance and knowledge levels (MacPhillamy et al., 2021). The para-veterinarians in Cambodia are the Village Animal Health Workers (VAHW) system that was established in early 1990, involving the selection of a local livestock-interested farmer to receive some training in basic village-level animal health services provision for smallholder farmers, particularly the vaccination of cattle for haemorrhagic septicaemia (HS) in rural communities (MacPhillamy et al., 2019). Similar approaches have been adopted and implemented in many developing countries, including Afghanistan (Leyland, 1993), Kenya (Blakeway, 1993; Mugunieri, Irungu, \& Omiti, 2004), Ethiopia (Admassu et al., 2005) and Nepal (Moktan, Mitchelhill, \& Joshi, 1990) and have shared similar purposes and goals.

The Department of Animal Health and Production (DAHP); now General Directorate of Animal Health \& Production (GDAHP) aimed to have one VAHW per village in every province across the country. The VAHW selection process in Cambodia required basic literacy, experience with livestock care and husbandry, and a willingness to learn and obtain qualifications. They were often a part-time local animal health service provider, supported in part by providing farm labour or with a little help from the community. The DAHP commenced VAHW training in the 1990s, with initial support from Non-Government Organisations (NGOs) and the Food and Agriculture Organisation of the United Nations (FAO) (Calba et al., 2014). VAHWs received basic training of one-month duration in animal health and a starting kit from the training providers, enabling the provision of fee-based basic animal health services to smallholder farmers, including treatments and vaccinations within their village. The VAHWs usually purchase veterinary drugs and related equipment from local veterinary drugstores and suppliers at the district or provincial level.

One of the important roles for the VAHWs is to contribute to government disease control programs by reporting disease outbreaks, especially FMD, to the District Office of Animal Health and Production (DOAHP). Further, VAHWs should assist in diagnostic investigations and volunteer their participation in the national vaccination campaigns, particularly against Haemorrhagic Septicaemia (HS) and FMD, as required. The 
VAHWs have been described as an important local resource, enabling persons with some animal health skills to assist smallholder farmers (Tornimbene, Chhim, Sorn, Drew, \& Guitian, 2014). They may also provide an important role in information exchange between local veterinarians and the community, particularly in improving biosecurity to prevent highly pathogenic avian influenza (HPAI) (Bhandari, Wollen, \& Lohani, 2011). The VAHWs were considered highly accessible, available, affordable, and trustworthy relative to other service providers (Admassu et al., 2005; Peeling \& Holden, 2004). Most VAHWs work closely with local authorities at village, commune, and district levels, especially during national vaccination campaigns (Government officials, personal communication, May 24, 2014).

Although the VAHWs was intended to provide local animal health services and assistance to local veterinary authorities in livestock disease prevention programs in their communities, their contributions to these tasks was uncertain (Stratton et al., 2015). A cross-sectional survey of VAHWs $(\mathrm{n}=445)$ from 19 provinces in Cambodia in 2008, used 'guided group interviews', to examine the roles of VAHWs and found they had good contact with farmers, with $61.5 \%$ making more than one farm visit daily, yet incomes from these services were low (Stratton et al., 2015). In 2015, a closed-ended cross-sectional study of VAHWs $(\mathrm{n}=80)$ from two provinces (Takeo and Tbong Khmum provinces) found positive associations between income generated from VAHW activities $(\mathrm{p}=.01)$ and the frequency of visiting farmers $(\mathrm{p}<.0001)$, although almost $90 \%$ of VAHW reported that they use antibiotics to treat FMD affected animals (MacPhillamy et al., 2019), potentially contributing to antimicrobial resistance risks (AMR).

In Cambodia, the severe socioeconomic impacts from FMD suggest an appropriate, sustainable national FMD control program is required (Blacksell et al., 2019; Young, Suon, Andrews, Henry, \& Windsor, 2013a; Young et al., 2016). However, due to the absence of effective biosecurity and the necessary emergency disease management and control measures, including the routine culling of infected animals and restrictions on the movement of animals and animal products, vaccination is becoming increasingly appropriate to assist in the control of FMD. However, the vaccination coverage of the national large ruminant population against FMD in the nationwide epidemic in 2010 was estimated at 2.7\%, compared with $43.8 \%$ for HS (Young et al., 2013a). The low adoption of FMD vaccination has continued with only $170,000(5.3 \%)$ and 267,000 (8.3\%) doses of FMD vaccine were imported (GDAHP and Private sectors) and used in 2019 and 2020 (cattle and buffalo population is 3,225,147 heads) compared to 2,015,000 (64.5\%) and 1,790,000 (55.5\%) doses of HS vaccine, respectively (H. Ponnaka, personal communication, November 18, 2020). It is readily apparent that preventive strategies for FMD control are mainly absent in Cambodia, and even in areas where vaccination has been applied, it has been insufficient to prevent ongoing FMD outbreaks (Sieng \& Kerr, 2013) .

There is currently no evidence that VAHWs have been privately providing FMD vaccination services to their own and farmers' animals within the communities. This objective of this study was to further assess the knowledge, attitudes, and practices (KAP) including the involvement of VAHWs in the provision of animal health services and FMD in particular, including the identification of potential opportunities for more engagement of VAHWs in provision of private vaccination services in their communities. This information may provide insights that could advise and assist animal health policy-makers in improving national livestock disease control programs in the country and TAD in the wider SEA region.

\section{Materials and methods}

\subsection{Study areas}

Both primary and secondary data were collected in this study. The primary data was derived from a detailed survey conducted in the two provinces of Kampong Cham (KC) and Pursat (PS). These provinces were selected for this study as both participated in the 'Domestic and International market development for high-value cattle and beef in South-East Cambodia' project (Figure 1). Pursat is located in the western part of the country and bordered with 4 provinces, Tonle Sap (Sap River) and Thailand. Kampong Cham is located on the central lowlands of the Mekong River and bordered to six provinces, Mekong River and Vietnam. The project was a collaborative research effort facilitated by the University of New England and DAHP, with funding by the Australian Centre for International Agricultural Research (ACIAR project 
$\mathrm{AH} / 2012 / 046)$.

$>$ Insert figure $1<$

The secondary data was collected during a nationwide review of the annual reports on VAHW programs, provided from the GDAHP, Ministry of Agriculture, Forestry and Fisheries, and included personal communications with the chiefs and vice chiefs of the Provincial Office of Animal Health and Production (POAHP) involved in the refreshing training and monitoring of the VAHW programs. The VAHW records were examined to provided temporal information on trends in the total number of VAHWs, their gender and their classification as 'active' or otherwise, in each province between 2011 to 2020. Interviews were also conducted with the Chief of POAHP in the 5 provinces where there was a high 'drop out' rate of VAHWs to provide information on their perceptions of the reasons for the cessation of VAWH activities.

\subsection{Data collection methods}

The VAHWs targeted for the primary data survey were identified and selected through informal discussions and consultations between the researcher, representatives of the POAHP, DAHP and the ACIAR-funded project team. The participating VAHWs were selected based on their active animal health services during the past six months and recommendations from the POAHP. Primary data was collected from random sample of VAHWs $(n=198)$ from PS $(n=113)$ and KC $(n=85)$. A semi-structured questionnaire consisting of open and closed questions was developed to collect quantitative and qualitative information from the VAHWs. The questionnaires were initially developed in English and then translated into Khmer by the senior author, ensuring all questions were easy to understand and answer. The questionnaire collected information on the background of VAHWs, household assets, household incomes, knowledge and experiences of vaccination and diseases, animal health services provided to both his own and farmers' livestock, plus their perceptions on the importance of vaccination. In addition to the specific questions, information on past vaccination programs' obstacles and opportunities were requested, and suggestions for improving vaccination programs were obtained. Before the survey, the survey team received a full day of training to ensure that they had a clear understanding of the study's aims and objectives and were confident in their roles.

The questionnaire and delivery technique was tested on three VAHWs in PS to evaluate suitability and clarity. After initial field testing, the questions were reviewed and modified by the team. Due to time and budget limitations, a guided group discussion method involving $20-25$ VAHWs lasted 2 to 3 hours. At least two or three enumerators were deployed at each guided group discussion to ensure they understood and answered the questions. Every VAHW received a questionnaire in Khmer, and the author or enumerators conducted a guided group discussion in Khmer (Figure 2). The enumerators or author read out the question (question by question) and explained the nature of the response/s requested. All VAHWs were then given a reasonable time to contemplate before answering each question. Enumerators ensured that they did not move too quickly between questions, the VAHWs were requested to respond to open questions by writing down truthful answers based on their experiences, and it was emphasised that the completed questionnaires were anonymous with no names were recorded. The VAHWs were advised not to share answers with their peers. Besides, during the group interviews, government officials were excluded from the interview to ensure confidentiality and encourage honest responses. At the end of the session, all completed questionnaires were collected and checked to ensure that no information was missing. The guided group discussions were conducted at each meeting in February and March 2014 (Figure 2).

\section{$>$ Insert figure $2<$}

The nationwide review of the annual reports on VAHWs from the GDAHP, Ministry of Agriculture, Forestry and Fisheries (MAFF), and personal communications with the chief and vice chiefs of the POAHP involved in the refreshing training and monitoring of the VAHW programs, provided the secondary data. The VAHW national records, including the total number of VAHW and gender in each province between 2011 to 2020 and 'active' classification of VAHWs between 2017 to 2020 in the annual reports, were summarised and analysed. The reasons for the cessation of the VAHW activities and the low rate of female VAHW were also obtained. The criteria used to determine whether a VAHW was active was agreed upon in interviews with 
the Chiefs of the 5 POAHP, conducted by telephone on December 16, 2020. Active VAWHs were those who: (1) had good knowledge of animal health and production and trust by farmers; (2) collaborated with and assisted the district/provincial veterinary authorities when needed; (3) providing good animal health and production services to farmers; and (4) permanently lived in the village.

\subsection{Data management and analysis}

The primary data were coded and transcribed into a database, and statistical analysis was performed using SPSS version 21.0 (SPSS Inc., IL, USA). Standard descriptive analyses of the data were performed with proportions of categorical variables. Investigation of variables considered to be probably associated with FMD vaccination practice was performed. Statistical analyses were undertaken by application of a chisquare test to possible associations between age group ( $1=22-40$ years old; $2=>40$ years old), education ( $1=$ high school; $2=$ secondary school; $3=$ primary school), annual household income $(1=<\mathrm{USD} 1,000 ; 2=\mathrm{USD}$ $1,000$ - USD 2,$000 ; 3=>$ USD 2,000$)$, number of years working as VAHW $(1=<6$ years; $2=6-10$ years; $3=>10$ years), and number of villages covered ( $1=$ only one village; $2=2-5$ villages; $3=>5$ villages $)$ with the FMD vaccination practice, specifically reported by VAHWs $(1=$ yes; $2=$ no).

The relevant VAHW data (secondary data) from the annual national reports were translated from Khmer into English, entered into Microsoft Excel (365), and then exported into SPSS for data analysis. Statistical analysis was performed using SPSS version 21.0. Descriptive statistics were used to describe the characteristics of the VAHWs.

\section{Results}

\subsection{Characteristics of VAHW participants}

Due to time and resource constraints, a total of 198 VAHWs participated in the survey, with $43 \%(\mathrm{n}=85)$ from Prey Chhor district in KC, and 14\% $(\mathrm{n}=27)$ from Sampov Meas district plus 39\% ( $\mathrm{n}=78)$ from Bakan district, both in PS. Eight VAHWs (4\%) from neighbouring villages in Korn Dieng district in PS also participated in the study. The majority of VAHWs were males $(91.4 \%, \mathrm{n}=181$ ) (Table 1) of mean ages 43 $(\mathrm{SD}=9.32)$ and $47(\mathrm{SD}=8.44)$ in $\mathrm{KC}$ and $\mathrm{PS}$, respectively.

$>$ Insert table $1<$

This indicated a clear male-domination of local animal health services in these rural communities. The male VAHWs were asked to explain why few female villagers sought this job, with responses on contributions to low female VAHW numbers including fear of travelling alone within the community $(89 \%, \mathrm{n}=161)$; culture and tradition forcing women to manage household affairs $(86 \%, \mathrm{n}=156)$; low confidence in working with large animals $(80 \%, \mathrm{n}=145)$; and lack of communication skills $(76 \%, \mathrm{n}=138)$. Most of the VAHWs in the study areas had a better education in comparison to other farmers within the villages. All of the 198 VAHWs participating in this study responded to the question of education. Of these, $28 \%(\mathrm{n}=55)$ and $34 \%(\mathrm{n}=67)$ had completed primary school, $42 \%(\mathrm{n}=83)$ and $51 \%(\mathrm{n}=101)$ secondary, and $25 \%(\mathrm{n}=49)$ and $13 \%(\mathrm{n}=$ 26) high school in KC and PS, respectively. Only six VAHWs in both study provinces had completed tertiary education. In addition to their role as VAHWs, most participants in both study areas $(89 \%, \mathrm{n}=176))$ were directly involved in farming activities. A small proportion of the VAHWs were also government officials or managed small businesses. Two-thirds of the VAHWs worked mainly with large ruminants (cattle) rather than small animals and poultry. It was noted that demand for animal health services for small animals and poultry was very low, with farmers considering treating these animals as unjustified.

\subsection{Household assets and financial situation}

The majority of VAHWs owned rice fields. The mean average area in PS was 3.0 ha (SD $=0.9$ ha) compared to 1.1 ha $(\mathrm{SD}=0.8 \mathrm{ha}$ ) in KC. One-quarter of VHAWs in KC and half in PS owned land for other crops (1.0 and 0.7 ha). VAHWs in PS owned slightly more village chickens $(33 ; \mathrm{SD}=31)$ and ducks $(25 ; \mathrm{SD}=54)$ on average than VAHWs in KC. The VAHWs in KC owned slightly more pigs $(6.9$; SD $=8)$ on average than VAHWs in PS. VAHWs owned an average of 4 cattle $(\mathrm{SD}=3)$ in both study areas. Of 85 and 113 VAHWs, 
$6 \%(\mathrm{n}=5)$ and $18 \%(\mathrm{n}=20)$ reported they had not owned any cattle in KC and PS, respectively. As only 10 VAHWs in PS had raised buffalo with cattle, buffalo were excluded from the study. Nearly one-third of VAHWs in KC had borrowed money $(31 \%, \mathrm{n}=26)$ in 2013, although the proportion was higher in PS (53\%, $\mathrm{n}=60)$. The majority of VAHWs not borrowing money stated $(90 \%, \mathrm{n}=53)$ in $\mathrm{KC}$ and $(87 \%, \mathrm{n}=46)$ in PS that they had no need, with a small proportion mentioning that high-interest rates and the unwillingness of moneylenders to provide a loan were their primary reasons. Of the VAHWs borrowing money, less than $20 \%$ used the money to buy animals, with $19 \%$ and $8 \%$ using the money to buy veterinary equipment, drugs, and vaccines in $\mathrm{KC}$ and $\mathrm{PS}$, respectively. The purchase of agricultural materials was an important reason for most VAHWs in KC (42\%) and PS (60\%) with other purposes, as reported by VAHWs, summarised in Table 2 .

$>$ Insert table $2<$

\subsection{Training and experiences}

The majority of VAHWs were trained by the technical staff from the POAHP (74\%), with the remaining participants trained by technical staff from DAHP (22\%) and Non-Government Organisations (NGOs). During their time as VAHWs, $67 \%(\mathrm{n}=57)$ in $\mathrm{KC}$ and $89 \%(\mathrm{n}=101)$ in PS had received refresher training on animal health and production. The participating VAHWs had provided animal health services to livestock smallholder farmers for an average of $8.4(\mathrm{SD}=4.89)$ and 11.2 years $(\mathrm{SD}=6.15)$ in $\mathrm{KC}$ and PS, respectively. Many VAHWs in both study provinces had a good level of experience, with $40 \%$ ( $\mathrm{n}=79$ ) having more than a decade. The majority of the VAHWs admitted that they enjoy their work as local animal health service providers in their communities.

\subsection{Sources of household income in 2013}

In general, the incomes of VAHW households varied between the study areas. The mean annual household income in 2013 was USD 1,627 (USD 75 - USD 8,110, SD = 1,401.8) in KC and USD 1,593 (USD 50 - USD $7,050, \mathrm{SD}=1,269.5$ ) in PS. The total income of the VAHW household was sourced from 5 main categories: agricultural cropping (rice and other crops), off-farm employment, sale of animals, animal health services, and vaccination services. Rice and other crops were the dominant income source in KC (41\%) and PS (60\%). The remaining income sources in KC and PS were off-farm employment $(21 \% ; 11 \%)$, sale of animals $(17 \% ; 16 \%)$, animal health services $(20 \% ; 9 \%)$, and vaccination services $(1 \% ; 4 \%)$. Thus the proportion of VAHW annual household income generated from their provision of animal health services was found to be approximately $21 \%$ and $13 \%$ in $\mathrm{KC}$ and PS, respectively.

\subsection{FMD vaccination experiences and practices}

The majority of VAHW $(96 \%, \mathrm{n}=190)$ stated that the primary purpose of vaccination is to protect animals against preventable diseases. However, approximately one-third of the VAHWs $(34 \%, \mathrm{n}=67)$ also believed that vaccination helped the animal gain weight and grow faster, and one-quarter of VAHWs $(25 \%, \mathrm{n}=49)$ thought vaccination could help treat sick animals. Nearly all surveyed VAHWs believed that only healthy animals should receive the vaccination. When asked for vaccination services on their animals during the last three years (2011 - 2013), most of them reported vaccinating their animals against HS, while only one-third vaccinated their animals against FMD in 2013 (Figure 3).

\section{$>$ Insert figure $3<$}

The study identified that there were no private HS and FMD vaccination services provided in their communities. The majority of VAHWs reported that HS and FMD vaccination programs were conducted through the government-subsidised vaccination program, and only some animals in each household herd were vaccinated against FMD and HS during the vaccination day. Approximately two-thirds of VAHWs $(64 \%, \mathrm{n}=56)$ reported that more than half of the household herd was vaccinated against HS, whereas only $27 \%$ ( $\mathrm{n}=17$ ) reported the herd was vaccinated against FMD in KC. In PS, $72 \%(\mathrm{n}=80)$ of VAHWs acknowledged that more than $50 \%$ of the household herd was vaccinated against HS, whereas only $15 \%(\mathrm{n}=7)$ of the herds were vaccinated against FMD. All VAHWs reported that DAHP/POAHP provided FMD vaccines, and they 
were asked to provide vaccination services during the vaccination campaign (80\%). Interestingly, when asked if they have never vaccinated their animals against HS and FMD during the three years, the study found that $10 \%(\mathrm{n}=8)$ and $49 \%(\mathrm{n}=39)$ of VAHWs in $\mathrm{KC}(\mathrm{n}=80)$ and $8 \%(\mathrm{n}=7)$ and $60 \%(\mathrm{n}=56)$ in PS $(\mathrm{n}=93)$, respectively, admitted that they had not. Even though VAHWs are local animal health service providers, none of them had vaccinated their animals every six months. Over three-quarters of VAHWs in both study areas had vaccinated their animals against HS once every year during the three years, while only one-third and almost half had vaccinated their animals against FMD in KC and PS, respectively (Figure 4).

$>$ Insert figure $4<$

The main reasons for not re-vaccinating their animals every six months, nearly one-third $(29 \%, \mathrm{n}=21)$ of VAHW in $\mathrm{KC}$ and nearly half $(44 \%, \mathrm{n}=38)$ in PS, refused to answer the question. Ranking of not re-vaccinating animals every six months by reason (from 1 to 3 with one as most important reason and three least important reason) found that the most common reason was the availability of vaccine (KC $49 \%, \mathrm{n}=35$; PS $35 \%, \mathrm{n}=30$ ), followed by the assumption that one vaccination every 12 months was sufficient to protect the animals ( $\mathrm{KC} 15 \%, \mathrm{n}=11$; PS $17 \%, \mathrm{n}=15)$. The high price of $\mathrm{FMD}$ vaccine, availability of vaccines only during the outbreak, the waiting for free FMD vaccination were also ranked as primary reasons by the minority of VAHWs. Nearly all cattle in the age group $>3$ years old and $<1$ year old in both study provinces were vaccinated against HS. Nearly one-third of cattle in the age group $>3$ years old were vaccinated against FMD, while a lower proportion of younger age group of $1-3$ years old and $<1$ year old have received the vaccination (Figure 5).

$>$ Insert figure $5<$

Four main activities were identified during the provision of the service by a VAHW, including providing animal health and husbandry advice during the farmer household visits and the village meetings, animal treatments, and vaccination. The relationships and responsibilities between various stakeholders such as veterinary authorities, local authorities, projects, vaccine suppliers, animal health service providers, and smallholder farmers are illustrated (Figure 6).

$>$ Insert Figure $6<$

Well over half of the VAHWs $(81 \%, \mathrm{n}=160)$ interviewed, reported that they provided animal health services to large ruminants, $14 \%(\mathrm{n}=28)$ to pigs and $6 \%(\mathrm{n}=12)$ to poultry more than $50 \%$ of their animal services. Above $60 \%(\mathrm{n}=119)$ of VAHWs in both study areas provided their animal health services in their villages and other villages $(2-5$ villages $)$, while the other $25 \%(\mathrm{n}=50)$ provided services within their village. The remaining $15 \%(\mathrm{n}=30)$ of VAHWs provided animal health services to more than five villages. Most of VAHWs (90\%) acknowledged that other VAHWs also came to offer general animal health services in their villages.

\subsection{Association with FMD vaccination practices}

Among five variables examined, there was no evidence of a relationship between FMD vaccination practice by the VAHW and education $(\mathrm{P}=0.141)$, annual household income $(\mathrm{P}=0.202)$, the number of years working as VAHW $(\mathrm{P}=0.524)$, and the number of villages covered $(\mathrm{P}=0.490)$. However, there was evidence of relationships between the age group of VAHW and the FMD vaccination practised $\left(X^{2}=4.321, \mathrm{df}=1, \mathrm{P}\right.$ $=0.041)$. In this case, for FMD vaccination practices, older VAHWs were more likely to advocate for FMD vaccination when vaccines were available from the veterinary authorities.

3.7 Vaccine cold storage and administration experiences and practices

Nearly all VAHWs reported that a cold box was an essential means of storing vaccines. Alternatively, only a few VAHWs living close to the district and provincial town were able to access electricity and use a refrigerator to store vaccines with other foods and drinks. Many VAWHs reported storing vaccines by using the cold box at neighbouring households. Approximately one-third of VAHWs reported that they were the only person in the family responsible for the vaccine cold box, with the remaining participants not 
answering this question. However, no VAHWs used a thermometer to monitor the temperature in the cold box. About two-thirds of VAHWs were confident in agreeing that a temperature range of between $2-8^{\circ} \mathrm{C}$ was an appropriate range for vaccines, with the remaining VAHWs considering that under $2^{\circ} \mathrm{C}$ and above $8^{\circ} \mathrm{C}$ were the suitable temperature ranges for storage of vaccines. All VAHWs were asked the question, "did VAHWs ever get reports of adverse reactions in vaccination programs in 2013 ?" and over $70 \%(\mathrm{n}=60)$ in $\mathrm{KC}$ and $50 \%(\mathrm{n}=57)$ in PS advised having received complaints from farmers. Of these, $79 \%(\mathrm{n}=156)$ in both provinces received less than three complaints from smallholder farmers in 2013, with the remainder having more than three in the same period. A lump at the inoculation site was the most common reason for the complaint identified by smallholder farmers, followed by inappetence.

In response to questions on HS and FMD vaccination costs, the majority of the VAHWs indicated that private vaccination was nonexistent in the study areas. However, they believed that the cost of FMD vaccination per head would be USD 1.3 ( $\mathrm{SD}=1.6)$ for HS and USD 2.1 ( $\mathrm{SD}=1.9)$ for FMD if the private sector provided vaccination services. The majority of VAHWs revealed that the actual fee that they received from the farmers during FMD vaccination service through government-subsidised HS and FMD programs was USD $0.2(\mathrm{SD}=0.1)$ per head. The POAHP arranged this fee to help cover the operational cost of the vaccination program. The mean recovery period for FMD was reported to vary from 9 days $(\mathrm{SD}=7.2)$ to 23 days $(\mathrm{SD}=20.8)$. The average cost for treating FMD infected animals was estimated at between USD 18.7 $(\mathrm{SD}=13.1)$ to USD $34.7(\mathrm{SD}=25.7)$ per head. The majority believed that the possibility of recovery rate was higher in adults (80\%) than growing steers or heifers (50\%). Nearly three-quarters of VAHWs advised they had not received any training or information on the impacts of losses due to FMD or the economic benefits of control programs, with the remaining VAHWs not responding to this question.

\subsection{Number of VAHWs reported by the POAHP}

The number of villages and VAHWs stratified by year and province between 2011 and 2020 at 10 year intervals is displayed (Table 3). There were considerable variations in the total number of VAHWs distributed in each province, including the number of female and percentage VAHWs between 25 provinces and municipality and the total number of VAHWs in the country between 2011 and 2020. The number of VAHWs in Thbong Khum province is of interest as this province was formed when Kampong Cham province was split in two by a Royal Degree on $31^{\text {st }}$ December 2013. Thus the number of VAHWs in Tbong Khmum was zero prior to 2014. It is also notable that the number of VAHWs decreased in 18 provinces $(72 \%)$ between $3 \%-72 \%$, increased in 4 provinces (16\%) between 1.5\% - 63\% and remained the same in 3 provinces (12\%) in 2020 when compared to 2019. These findings indicate that there has been a considerable decline in the number of VAHWs in the most recent two years, and is a concerning trend. The proportion of female VAHWs was consistently low throughout the period of 10 years between 2011 to 2020 , with the mean at $8.26 \%$ (SD = 1.019). The reasons provided as to why females did not want to become an VAHW, was investigated with the chiefs and vice chiefs of 15 POAHPs, including two female chiefs. They reported the reasons as follows: low confidence in working with large ruminants $(56.3 \%-78.4 \%)$; travel, personal security and safety concerns $(52.3 \%$ - $69.6 \%)$; Khmer cultural issues $(40.4 \%$ - $55.6 \%)$; and negative influences from family members $(12.4 \%$ $-19.4 \%)$.

$>$ Insert Table $3<$

The VAHWs records indicated that the total number of VAHWs fell slightly from 12,420 in 2017 to 11,786 in 2018, 11,747 in 2019, and then dropped to 8,920 in 2020. The total number of VAHWs in 2020 declined by $28.2 \%, 24.4 \%$ and $24.1 \%$ compared to 2017,2018 and 2019 , respectively. The number of females was consistently very low, declining from 1,039 (8.4\%) in 2017, to 971 (8.3\%), $927(7.9 \%)$ and $581(6.2 \%)$ in 2018, 2019 and 2020, respectively. The number of VAHWs considered as active, was also consistently low, at 5,328 (42.7\%), 5,326 (45.0\%), 4,150 (39.0\%) and 4,915 (55.1\%) in 2017, 2018, 2019 and 2020, respectively (although it is noted that the POAHP only commenced recording the number of active VAHW from 2017). The mean proportion of female VAHW $(6.45 \%$ - $8.41 \%)$ and active VAHW $(40.25 \%$ - $55.66 \%)$ in each province and between 2017 and 2020 is provided (Table 4). The low proportions of both active and female VAHWs reported by the POAHP throughout the last 4 years (between 2017 to 2020) are of concerns, particularly as 
males remain the dominate gender of the majority of VAHWs in the country during the past 10 years.

$>$ Insert Table $4<$

Following examination of the VAHW records, 5 provinces with the highest 'dropout' rate were selected for further analysis. In 2020, the proportion of VAHWs had dropped by $67.5 \%, 66.7 \%, 57.6 \%, 43.5 \%$ and $35.9 \%$ in Mondulkiri, Kandal, Siem Reap, Kampong Cham and Battambang provinces when compared to 2019, respectively. The most common reasons why VAHWs ceased their activities in 2020 is displayed (Figure 7). An investigation of the reasons why so many VAHWs ceased their activities in these 5 provinces, the chiefs of the POAHP reported the reasons as follows: they did not want to continue their jobs as local animal health services provider $(52.1 \%-71.5 \%)$; migration (14.3\% - 38.4\%); home relocation $(0 \%-15.9 \%)$; and increasing age or death $(1.7 \%-8.9 \%)$ (Figure 7$)$.

$>$ Insert Figure $7<$

3.8 Perceptions of VAHWs on importance of diseases and vaccination

VAHWs were perceived to have an essential role in assisting the DOAHP/POAHP in the provision of primary animal health services to smallholder farmers in their communities. In considering the effectiveness of past vaccination programs, four possible factors for effectiveness were identified and ranked from very important to not important. Most VAHWs acknowledged that splitting doses of vaccine, poor quality of vaccines, poor crush sites, incorrect storage of vaccines, and prolonged vaccination times are very important factors for vaccination success in both studies areas. The majority of VAHWs believed inadequate knowledge of diseases and vaccination $(92 \%, \mathrm{n}=182)$ by farmers was an important factor preventing them from participating in the vaccination programs, followed by poor vaccination campaigns administration and lack of accessibility to FMD vaccines (Table 5). The majority of VAHWs $(87 \%, \mathrm{n}=172)$ believed that improvement in the knowledge of local authorities, especially village and commune chief on animal diseases and vaccination, was a very important factor that can be instrumental in improving future vaccination rates in their communities. Other factors influencing the uptake of vaccination by farmers included improved coordination and cooperation between local authorities and the district and provincial veterinarians, followed by improved communications enabling raising important issues in every village meeting with farmers. Most VAHWs advised that the POAHP/DOAHP should have appropriate planning, better awareness programs, and good training programs for farmers. Most VAHWs believed that the most important factors to improve the effectiveness of vaccination programs were the need to establish appropriate vaccination planning, using only good quality vaccines, working with different stakeholders to get more farmer participation, and establishing appropriate crush sites for more efficient animal restraint.

$>$ Insert table $5<$

\section{Discussion}

The guided group discussion survey of VAHWs $(n=198)$ in the two study provinces of Kampong Cham and Pursat in Cambodia provides details of their KAP's on animal health and disease prevention programs in their communities. The study found that the number of female VAHWs participation in the survey was very low (8\%) although this was consistent with the reported average percentage of female VAHWs in 2010 (8.4\%) (Stratton et al., 2015), 2015 (7.7\%) and 2020 (6.2\%) (MAFF, 2017, 2020). The mean proportion of female VAHWs in the country during the 10 years between 2011 and 2020 was $8.26 \%$ (SD = 1.019). The investigation with the chiefs and vice chiefs of the ten provinces, suggested that the low female participation rate as VAHWs was due to three main reasons: low confidence in working with large ruminants; travel, personal security and safety concerns; and Khmer cultural issues. These three responses provided by the chiefs and vice chiefs of the POAHP were consistent with those provided in interviews with the 198 VAHWs, including 17 female VAHWs.

The study identified that despite VAHWs providing local animal health services in these provinces, the annual household incomes from these activities were not well rewarded, contributing only an average of $21 \%$ (KC) and $13 \%$ (PS) of the total household annual income in 2013. Crop cultivation, off-farm employment, and the 
sale of animals as income sources were shown to be more important for VAHW incomes. This is consistent with a previous study from 2008 that identified most VAHWs (66\%) from 19 provinces earned less than $40 \%$ of their household income from animal health and vaccination services (Stratton et al., 2015). However, a 2015 survey of VAHWs from Takeo and Tbong Khmum provinces identified that although incomes from VAHW activities were also generally low with monthly incomes predominantly within the USD 48.80 - USD 97.50 income bracket (38\%), $62 \%$ of VAHW earning over USD 97.50/month had over 10 years of VAHW experience, and there also appeared to be a strong association between income generating activities and frequency of contact with district animal health authorities (MacPhillamy et al., 2019).

The survey data identified that many VAHWs cease their activities for various reasons, including the low income generated from the provision of local animal health services. Information from the GDAHP annual reports indicated that nationwide, the number of VAHWs had decreased in $2020(8,920)$ by $28 \%$ and $27 \%$ compared to records from $2011(14,861)$ and $2015(12,221)$, respectively. The number of VAHWs continues to decline in many provinces in the coming year (POAHP, personal communication, December 16, 2020). Despite the decrease in the total number of VAHWs, the recent records indicate that not all VAHWs had been providing active animal health services and assistance to the veterinary authorities at the district and provincial levels. The mean of active VAHW in the country for the last 4 years (2017 - 2020) was $46 \%$ $(\mathrm{SD}=6.735)$. Personal communication with chiefs and vice chiefs of the five provinces with higher dropout rates of VAHWs, suggested that resignation or cessation of VAHW activities due to low incomes is the most important reason, followed by migration, house relocation, and increasing age or death. For the sustainability of the VAHW program, this suggests that increasing the roles for VAHWs in their communities is required, enabling them to earn a more reasonable and sustainable income to support their families.

The majority of VAHWs provided their animal health services to large ruminants rather than pigs or poultry, and these findings were similar to the previous reports from Cambodia (Bhandari et al., 2011; MacPhillamy et al., 2019; Stratton et al., 2015) and northern Malawi (Huttner, Leidl, Jere, \& Pfeiffer, 2000). It should be noted that although VAHWs may wish to provide animal health services to poultry, farmers generally do not think it worthwhile spending money for low-value village poultry. The study also found that $75 \%$ of VAHWs provided animal health services to the farmers in their village and other villages, generating more income for their households. This suggests that more effective VAHWs could receive more calls and generate more income from providing their services both within and between villages during the disease outbreaks. However, it is noted that frequent VAHWs travel and contact with different animals is a high-risk activity for disease transmission, requiring high standards of biosecurity practice. Currently, this knowledge of VAHWs remains limited. The Cambodian government is aware of the usefulness of having VAHWs serving their rural communities by delivering animal health services including treatment and vaccinations, but also potentially in the reporting of new disease outbreaks (cases) and contributing to a more effective national disease surveillance system. As recently suggested, refresher training on simple biosecurity measures for VAHWs is very important to enhance their preventive and biosecurity activities during household visits, and they can provide advice on this aspect to other villagers (MacPhillamy et al., 2019; MacPhillamy et al., 2021). The VAHWs during the interview displayed an understanding of FMD and the purpose of vaccination. However, the study revealed that they do not adequately vaccinate their own livestock against that important disease, with 50\% of VAHWs have never vaccinated their animals against FMD during 2011 - 2013. The application of Chi-Square test results indicated that in this survey, general education, annual household income, number of years working as VAHW, and number of villages covered, were not associated with FMD vaccination practices by VAHWs. This finding suggests that no matter how well educated, wealthy, hardworking, and experienced is a VAHW, this may not influence FMD vaccination uptake. However, the analysis did indicate that uptake of FMD vaccination was associated with the age group of VAHW.

It is likely that those VAHWs who never vaccinated their animals against FMD may not have received the FMD vaccine from the government-subsidised FMD vaccination program, or their villages were excluded from the government-subsidised FMD vaccination program. Low FMD vaccination rates as reported by VAHWs were due to limited access to FMD vaccine either at the POAHP or local veterinary drug stores and no private sector FMD vaccination service was provided in this survey area. As this preventive disease 
program was invariably due to vaccination for large ruminant provided for free (government-subsidised vaccination program) previously, it is likely that VAHWs and smallholders expected this to remain so. However, a minority of VAHWs may intend to provide, and smallholder farmers may intend to receive private FMD vaccination, although this is often difficult for them to organise as FMD vaccines are expensive and only available in vials of 50 doses. This requires the VAHWs to arrange and work with smallholder farmers seeking to have their animals vaccinated. It is also likely that VAHWs' unwillingness to organise private FMD vaccination within their own communities may be due to their perceived low benefits from providing this service, particularly as the treatment of sick animals with antimicrobials enables them to generate more income for the families. With limited understanding of the benefits of providing a whole of village 'fee for service' vaccination option, they may prefer to wait for the limited government-subsidised vaccination program to be delivered, with more opportunities to treat sick animals due to low vaccination rates, potentially could generate more household income for VAHWs.

This field survey conducted in both study provinces involved 198 VAHWs. There are several considerations associated with this group discussion survey. Firstly, the results present a snapshot of activities undertaken during three calendar years, and it is recognised that the performances of interviewed VAHWs will change over time. Secondly, for overall reported activities, such as treatment and vaccination, the data obtained may be considered representative for VAHWs in the whole province. Thirdly, there is always an issue with the accuracy of the response as interviewed VAHWs may be reluctant to provide correct and true information if they know it is against the existing government or social policy or norms.

This study from 2013 confirmed that as disease prevention programs relied on a limited budget from the veterinary authorities, subsidies for the FMD vaccination program were inadequate to provide effective immunological protection of the livestock population for FMD prevention or suppression. Despite increasing availability of FMD vaccine, this situation still exists in 2021.

Private FMD vaccination services provided by VAHWs and local veterinarians in the study area were nonexistent in 2013 and remain extremely sub-optimal in 2021. Unavailability of FMD vaccination services and lack of a reliable vaccine supply in the communities was identified as an important problem involving the absence of links and communication between vaccine demands from communities and vaccine supplies from vaccine retailers. The DAHP (now GDAHP) and POAHP should recognise this as an important issue that needs attention, as appropriate solutions to improve the participation of VAHWs in government and private disease prevention programs are required. Introducing information into short training programs for VAHWs and smallholder farmers on the importance of economic losses due to FMD and benefits from disease prevention is considered necessary to improve future vaccination uptake and disease control programs in Cambodia. The conclusion from the 2013 study confirmed that it is worthwhile for farmers to participate in the full-fee bi-annual FMD vaccination program if there are average annual outbreaks with a morbidity rate of approximately $30 \%$ and two major outbreaks with morbidity rate above $50 \%$, in the ensuing five years (Sieng, Patrick, Walkden-Brown, \& Sar, 2021b). This is consistent with conclusions from the 2015 survey that also suggested that improved training of VAHWs could ensure they remain important in supporting the under-resourced national veterinary services (MacPhillamy et al., 2021). In that study, binomial logistic regression modelling found the length of employment $(\mathrm{p}=.003)$, reporting of pig diseases $(\mathrm{p}=.007)$, recommending the quarantine of newly purchased animals $(\mathrm{p}=.008)$, treatment of cases of HS with antibiotics $(\mathrm{p}=.032)$ and the washing of footwear with water when entering or leaving a farm $(\mathrm{p}=.008)$, were all positively associated with a higher ([?]92\%) animal health and biosecurity knowledge score (MacPhillamy et al., 2021).

Increasing information is emerging on the importance of FMD vaccination and biosecurity in Cambodia and the importance of progressing this issue more effectively with VAHWs and smallholder farmers is necessary as this may contribute significantly to the improvement of the vaccination uptake by rural communities and assist the development of more effective disease control strategies in the country (MacPhillamy et al., 2019; MacPhillamy et al., 2021; Stratton et al., 2015; Young et al., 2013a; Young et al., 2017; Young et al., 2016). The veterinary authorities at the central and provincial levels need to focus their attention on 
several aspects of the vaccine supply chain, including provision of sufficient doses of high quality vaccines from reliable vaccine suppliers that are matched to currently circulating FMD serotypes. VAHWs need to be better informed on FMD vaccination and trained to understand that they could generate more income from protecting their animals and the majority of livestock within the communities from diseases. Further, they should contribute to informing smallholders on FMD prevention and ensuring that farmers learn to trust that regularly vaccinated animals that are fully protected is a far more effective and sustainable animal production system than the current situation, where a vulnerable population exists and the income of VAHW is largely derived from the expensive treatment of sick animals, potentially incurring AMR and food safety risk. Further, the lessons learned from delivery of large FMD vaccination programs in the Mekong region need greater awareness by all involved in TAD control in Cambodia and beyond (Blacksell et al., 2019; Nampanya, Khounsy, Abila, \& Windsor, 2018).

As this study was limited to two provinces involved in a defined research project, caution is advised in interpreting these findings as representative of the majority of Cambodia. This caution has been advised in the 2015 cross-sectional study that was also conducted within defined research project in two provinces (MacPhillamy et al., 2019), that examined disease reporting by VAHWs in Cambodia and found much lower contact between VAHW and district animal health authorities than recorded from the broader 2008 study in 19 provinces (Stratton et al., 2015). These variations in findings likely reflect spatial, temporal and methodological differences between the studies, particularly as in the 2008 study, the districts were randomly selected whilst the VAHWs were purposively selected. The 2015 study (MacPhillamy et al., 2019) and our 2013 study, purposively selected the study locations and used a probability sampling method to select VAHWs, with both techniques possibly introducing selection bias through increased contact of researchers with the VAHWs. Although the sample size of this 2013 study and the 2015 study is considerably smaller than that used in 2008, the studies do show that the issues identified here are similar across most provinces (MacPhillamy et al., 2019; MacPhillamy et al., 2021; Stratton et al., 2015).

Animal health extension approaches to improving disease control and biosecurity practices have been investigated in Cambodia, with participatory 'applied field research', 'on the job' training plus 'formal' training programmes found to encourage smallholder farmer motivations through nutritional interventions that improve the value of their cattle 'bank', offer better marketing opportunities and creating a more receptive environment for introduction of disease risk management for infectious and other production limiting diseases (Nampanya, Suon, Rast, \& Windsor, 2012). It was suggested that in lieu of a widespread public awareness programme to deliver mass education of smallholder farmers in disease prevention and biosecurity, livestock development projects in SE Asia should be conducting intensive training in disease risk management if trading in large ruminant livestock and large ruminant meat is to continue to progress and contribute to improve smallholder incomes and help address global food security concerns. Additional research is suggested to determine the best possible extension and public awareness activities and methods for encouraging VAHWs, smallholder farmers, and local authorities to be involved in livestock disease prevention programs, particularly if the development of sustainable private disease preventive programs is to be advocated.

Improvement of FMD control requires the cooperation of villagers, VAHWs, and village leaders in disease reporting, with either improved funding of government vaccination services and/or development of a private FMD vaccination service. Both strategies should be accompanied by training programs for farmers on disease transmission and the importance of biosecurity and vaccination, including information on the cost-benefits of treatment versus full fee bi-annual FMD vaccination (Sieng et al., 2021b; Sieng et al., 2021a). These initiatives are urgently required in Cambodia if the substantial increase in future FMD vaccination rates currently needed is to be achieved.

In conclusion, the findings from the field survey and desktop review of the contributions of the VAHWs to Cambodian livestock health services, indicate that the VAHW system is currently weak with a limited capacity to provide the level of services required to manage and control FMD in Cambodia. It is suggested that the GDAHP requires more effective policies that can strengthen the current VAHW system and improve the capacity for service delivery. The promotion of the VAHW role as a potential sustainable small business 
model through the sale of higher-level services to increasingly valuable livestock, is a suggested strategy. This strategy would enable higher retention rates of 'active' status VAHWs, potentially improving their contributions to disease reporting, surveillance and TAD control. These policies should also recognise the important issue of gender equity and encourage more women to become active VAHWs and potential leaders of local rural businesses. A critical component of the change management process required by all stakeholders involved in promotion of livestock development in Cambodia, is the development of gender sensitive training programs. These are urgently required if the currently weak VAHW system is to be strengthened and offer the required contributions to regional food security through enhanced livestock health and production, including TAD control.

\section{Acknowledgments}

The authors are grateful for funding by the Australian Government, delivered through the Australian Centre for International Agricultural Research, for the research project 'Domestic and International market development for high-value-cattle and beef in South-East Cambodia' (ACIAR AH/2010/046), that supported this study as part of a PhD studentship at the University of New England, Armidale, NSW, Australia. The authors also thank staff from the Department and Provincial Offices of Animal Health and Production staff for supporting the VAHW survey in the study areas, including respective project counterparts Soum Veoun and Lorn Sophal, graduate students from the Royal University of Agriculture Cambodia, independent assistant researchers, village chiefs, and the participating village animal health workers; their time, effort, kindness and sharing their hard work and attitude were essential to this study.

\section{Ethics standards}

Statement of human rights: Approval to use the questionnaire was obtained from the University of New England Human Research Ethics Committees (Approval No. HE 13-242, November 19, 2013 - November 19, 2014). Informed consent was obtained from all individual participants included in the study. This article does not contain any studies with animals performed by any of the authors.

\section{Conflict of interest statement}

The authors declare that they have no conflict of interest.

\section{References}

Admassu, B., Nega, S., Haile, T., Abera, B., Hussein, A., \& Catley, A. (2005). Impact assessment of a community-based animal health project in Dollo Ado and Dollo Bay districts, southern Ethiopia. Tropical animal health and production, 37 (1), 33-48.

Bhandari, D. P., Wollen, T. S., \& Lohani, M. N. (2011). Preventing highly pathogenic avian influenza (HPAI) at the rural community level: a case study from Cambodia. Tropical animal health and production, 43 (6), 1071-1073.

Blacksell, S. D., Siengsanan-Lamont, J., Kamolsiripichaiporn, S., Gleeson, L. J., \& Windsor, P. A. (2019). A history of FMD research and control programmes in Southeast Asia: lessons from the past informing the future. Epidemiology 83 Infection, 147 .

Blakeway, S. (1993). Animal health-community problem, community care. Appropriate Technology, 19 (4), $5-7$.

Calba, C., Ponsich, A., Nam, S., Collineau, L., Min, S., Thonnat, J., \& Goutard, F. L. (2014). Development of a participatory tool for the evaluation of Village Animal Health Workers in Cambodia. Acta tropica, 134 , 17-28.

Huttner, K., Leidl, K., Jere, F., \& Pfeiffer, D. (2000). Characteristics and performance of village animal health workers and veterinary assistants in northern Malawi.Journal of the South African Veterinary Association, 71 (3), p. 160-165. 
Leyland, T. (1993). Animal health care in Afghanistan. Appropriate Technology, 19 (4), 29-32.

MacPhillamy, I., Young, J., Siek, S., Bun, C., Suon, S., Toribio, J. A., . . . Bush, R. (2019). Improving Village Animal Health Worker participation in national disease surveillance systems: A case study from Cambodia. Transboundary and Emerging Diseases, 67 (2), 967-978.

MacPhillamy, I., Young, J., Vitou, S., Chanphalleap, H., Suon, S., Windsor, P., . . . Bush, R. (2021). Can improving animal health and biosecurity knowledge of para-veterinarians in Cambodiaassist in addressing challenges in smallholder livestock farming? Transboundary and Emerging Diseases .

MAFF. (2017). Annual report animal health and production in 2016 and direction for implementation in 2017. General Directorate of Animal Health and Production, Ministry of Agriculture, Forestry and Fisheries, Phnom Penh .

MAFF. (2020). Annual report animal health and production in 2019 and direction for implementation in 2020. General Directorate of Animal Health and Production, Ministry of Agriculture, Forestry and Fisheries, Phnom Penh .

Moktan, D., Mitchelhill, B., \& Joshi, Y. (1990). Village animal health workers in the Koshi hills: an evaluation report. PAC Working Paper-Pakhribas Agricultural Centre (4).

Mugunieri, G., Irungu, P., \& Omiti, J. (2004). Performance of community-based animal health workers in the delivery of livestock health services. Tropical animal health and production, 36 (6), 523-535.

Nampanya, S., Khounsy, S., Abila, R., \& Windsor, P. (2018). Implementing large Foot and Mouth Disease vaccination programmes for smallholder farmers: lessons from Lao PDR. Epidemiology $\& 3$ Infection, 146 (16), 2086-2095.

Nampanya, S., Suon, S., Rast, L., \& Windsor, P. (2012). Improvement in smallholder farmer knowledge of cattle production, health and biosecurity in Southern Cambodia between 2008-2010. In Cattle health, production and trade in Cambodia, ed. by Young J.R., Rast L., Suon S. and Windsor P.A. Paper presented at the Tropical animal health and production.

Peeling, D., \& Holden, S. (2004). The effectiveness of community-based animal health workers, for the poor, for communities and for public safety. Revue scientifique et technique-Office international des epizooties, 23 (1), 253-276.

Samkol, P., Sath, K., Patel, M., Windsor, P., \& Holtenius, K. (2015). Survey of smallholder beef cattle production systems in different agro-ecological zones of Cambodia. Tropical animal health and production, 7 (47), 1299-1306.

Sieng, S., \& Kerr, J. (2013). Investigation of vaccination effectiveness in two Cambodian villages facing an outbreak of foot-and-mouth disease. In Cattle health, production and trade in Cambodia, ed. by Young J.R., Rast L., Suon S. and Windsor P.A. , ACIAR Proceedings No. 138, 67-71.

Sieng, S., Patrick, I., Walkden-Brown, S., \& Sar, C. (2021b). A cost-benefit analysis of foot and mouth disease control program for smallholder cattle farmers in Cambodia. Authorea Preprints .

Sieng, S., Patrick, I., Windsor, P., Walkden-Brown, S., Sar, C., Smith, G., \& Kong, R. (2021a). Knowledge, attitudes, and practices of smallholder farmers on foot and mouth disease control in two Cambodian provinces. Authorea Preprints .

Stratton, J., Toribio, J., Suon, S., Young, J., Cowled, B., \& Windsor, P. (2015). Are Village Animal Health Workers Able to Assist in Strengthening Transboundary Animal Disease Control in Cambodia? Transboundary and Emerging Diseases .

Tornimbene, B., Chhim, V., Sorn, S., Drew, T., \& Guitian, J. (2014). Knowledge, attitudes and practices of Cambodian swine producers in relation to porcine reproductive and respiratory syndrome (PRRS). Preventive veterinary medicine, 116 (3), 252-267. 
Young, J., O'Reilly, R., Ashley, K., Suon, S., Leoung, I., Windsor, P., \& Bush, R. (2014b). Impacts on rural livelihoods in Cambodia following adoption of best practice health and husbandry interventions by smallholder cattle farmers. Transboundary and Emerging Diseases, 61 , 11-24.

Young, J., Rast, L., Suon, S., Bush, R., Henry, L., \& Windsor, P. (2014c). The impact of best practice health and husbandry interventions on smallholder cattle productivity in southern Cambodia. Animal Production Science, 54 (5), 629-637.

Young, J., Suon, S., Andrews, C., Henry, L., \& Windsor, P. (2013a). Assessment of financial impact of Foot and Mouth Disease on smallholder cattle farmers in southern Cambodia. Transboundary and Emerging Diseases, 60 , 166-174.

Young, J., Suon, S., Leoung, V., Kea, P., Hout, S., Thong, S., . . . Windsor, P. (2013b). Parasitic infections of large ruminants in Cambodia. In Cattle health, production and trade in Cambodia, ed. by Young J.R., Rast L., Suon S. and Windsor P.A., ACIAR Proceedings No. 138 , 53-58.

Young, J., Suon, S., Olmo, L., Bun, C., Hok, C., Ashley, K., . . . Windsor, P. (2017). Investigation of smallholder farmer biosecurity and implications for sustainable foot-and-mouth disease control in Cambodia. Transboundary and Emerging Diseases, 64 (6), 2000-2012.

Young, J., Suon, S., Rast, L., Nampanya, S., Windsor, P., \& Bush, R. (Producer). (2016). Benefit-Cost Analysis of Foot and Mouth Disease Control in Large Ruminants in Cambodia. Transboundary and Emerging Diseases.

\section{Hosted file}

VAHW-FMD-Figures 04052021.pdf available at https://authorea.com/users/401086/articles/521730contributions-of-village-animal-health-workers-to-foot-and-mouth-disease-control-incambodia

\section{Hosted file}

VAHW-FMD-Tables 04052021.pdf available at https://authorea.com/users/401086/articles/521730contributions-of-village-animal-health-workers-to-foot-and-mouth-disease-control-incambodia 